\title{
HOPF-GALOIS STRUCTURES ON FINITE EXTENSIONS WITH QUASISIMPLE GALOIS GROUP
}

\author{
CINDY (SIN YI) TSANG
}

\begin{abstract}
Let $L / K$ be a finite Galois extension of fields with Galois group $G$. It is known that $L / K$ admits exactly two Hopf-Galois structures when $G$ is non-abelian simple. In this paper, we extend this result to the case when $G$ is quasisimple.
\end{abstract}

\section{Contents}

1. Introduction 1

2. Crossed homomorphisms 3

3. Consequences of CFSG 5

4. Proof of Theorem $1.4 \quad 6$

4.1. Non-perfect groups $\quad 7$

4.2. Perfect groups 11

$\begin{array}{ll}\text { Acknowledgments } & 17\end{array}$

$\begin{array}{ll}\text { References } & 17\end{array}$

\section{INTRODUCTION}

Let $L / K$ be a finite Galois extension of fields with Galois group $G$. By [10], we know that each Hopf-Galois structure $\mathcal{H}$ on $L / K$ is associated to a group $N_{\mathcal{H}}$ of the same order as $G$. For each group $N$ of order $|G|$, define

$$
e(G, N)=\#\left\{\text { Hopf-Galois structures } \mathcal{H} \text { on } L / K \text { with } N_{\mathcal{H}} \simeq N\right\} .
$$

Let $\operatorname{Perm}(N)$ be the group of all permutations on $N$. Recall that a subgroup of $\operatorname{Perm}(N)$ is regular if its action on $N$ is regular. For example, clearly $\lambda(N)$ and $\rho(N)$ are regular subgroups of $\operatorname{Perm}(N)$, where

$$
\begin{cases}\lambda: N \longrightarrow \operatorname{Perm}(N) ; & \lambda(\eta)=(x \mapsto \eta x) \\ \rho: N \longrightarrow \operatorname{Perm}(N) ; & \rho(\eta)=\left(x \mapsto x \eta^{-1}\right)\end{cases}
$$

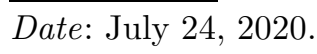


are the left and right regular representations of $N$. By work of [10] and [4], we have the formula

$$
e(G, N)=\frac{|\operatorname{Aut}(G)|}{|\operatorname{Aut}(N)|} \cdot \#\left\{\begin{array}{c}
\text { regular subgroups of } \operatorname{Hol}(N) \\
\text { which are isomorphic to } G
\end{array}\right\},
$$

where $\operatorname{Hol}(N)$ is the holomorph of $N$ and is defined to be

$$
\operatorname{Hol}(N)=\rho(N) \rtimes \operatorname{Aut}(N) .
$$

The calculation of $e(G, N)$ has been an active line of research because HopfGalois structures have application in Galois module theory; see [7] for more details. Let us also note in passing that regular subgroups of the holomorph are related to set-theoretic solutions to the Yang-Baxter equation; see [11].

For $N \simeq G$, the number $e(G, N)$ must be non-zero because $\lambda(N)$ and $\rho(N)$ are regular subgroups of $\operatorname{Hol}(N)$; note that $\lambda(N)$ and $\rho(N)$ are equal exactly when $N$ is abelian. For $N \nsucceq G$, the number $e(G, N)$ could very well be zero. In certain extreme cases, it might happen that

$$
e(G, N)= \begin{cases}1 & \text { for } N \simeq G \text { when } G \text { is abelian, } \\ 2 & \text { for } N \simeq G \text { when } G \text { is non-abelian, } \\ 0 & \text { for all other } N \nsucceq G .\end{cases}
$$

For $G$ abelian, by [4, Theorem 1] we know exactly when (1.1) occurs:

Theorem 1.1. If $G$ is a finite abelian group, then (1.1) holds precisely when the orders of $G$ and $(\mathbb{Z} /|G| \mathbb{Z})^{\times}$are coprime.

For $G$ non-abelian, the situation is more complicated. By $[5,6]$, we have:

Theorem 1.2. If $G$ is a finite non-abelian simple group, then (1.1) holds.

It is natural to ask whether Theorem 1.2 may be generalized to other nonabelian groups $G$ which are close to being simple. Recall that $G$ is said to be quasisimple if $G=[G, G]$ and $G / Z(G)$ is a simple group, where $[G, G]$ is the commutator subgroup and $Z(G)$ is the center of $G$. In [16, Theorem 1.3], the author has already shown that:

Theorem 1.3. If $G$ is a finite quasisimple group, then $e(G, G)=2$. 
HOPF-GALOIS STRUCTURES ON FINITE EXTENSIONS WITH QUASISIMPLE GALOIS GROUP 3

It remains to consider the groups $N \not f$. In [16, Theorem 1.6], the author has shown that if $G$ is the double cover of $A_{n}$ with $n \geq 5$, then $e(G, N)=0$ for all groups $N \not \subset G$ of order $n$ !. We shall extend this result and prove:

Theorem 1.4. If $G$ is a finite quasisimple group, then $e(G, N)=0$ for all groups $N \not G$ of order $|G|$.

In view of Theorems 1.3 and 1.4, one might guess that (1.1) is also true for say, finite almost simple or non-abelian characteristically simple groups $G$. If $G=S_{n}$ with $n \geq 5$, however, then by [6, Theorems 5 and 9], we have

$$
e(G, G) \neq 2 \text { and } e(G, N) \neq 0 \text { for some } N \not 千 G \text {. }
$$

See $[19,21]$ for generalizations to other finite almost simple groups $G$. If $G$ is a finite non-abelian characteristically simple group which is not simple, then $e(G, G) \neq 2$ by [17], but as far as the author knows, there is no investigation yet on whether there exists $N \not f G$ such that $e(G, N) \neq 0$.

\section{Crossed homomorphisms}

In this section, let $G$ and $\Gamma$ be finite groups, whose orders are not assumed to be equal. Given $\mathfrak{f} \in \operatorname{Hom}(G, \operatorname{Aut}(\Gamma))$, recall that a crossed homomorphism (with respect to $\mathfrak{f}$ ) is a map $\mathfrak{g}: G \longrightarrow \Gamma$ which satisfies

$$
\mathfrak{g}(\sigma \tau)=\mathfrak{g}(\sigma) \cdot \mathfrak{f}(\sigma)(\mathfrak{g}(\tau)) \text { for all } \sigma, \tau \in G .
$$

Let $Z_{\mathfrak{f}}^{1}(G, \Gamma)$ be the set of all such maps $\mathfrak{g}$. The regular subgroups of $\operatorname{Hol}(\Gamma)$ isomorphic to $G$ may be parametrized by the bijective maps in $Z_{\mathfrak{f}}^{1}(G, \Gamma)$.

Proposition 2.1. The regular subgroups of $\operatorname{Hol}(\Gamma)$ isomorphic to $G$ are precisely the sets $\{\rho(\mathfrak{g}(\sigma)) \cdot \mathfrak{f}(\sigma): \sigma \in G\}$, as $\mathfrak{f}$ ranges over $\operatorname{Hom}(G, \operatorname{Aut}(\Gamma))$ and $\mathfrak{g}$ over the bijective maps in $Z_{\mathfrak{f}}^{1}(G, \Gamma)$.

Proof. The proof is straightforward; see [16, Proposition 2.1].

Hence, when $\Gamma$ has order $|G|$, that $e(G, \Gamma)$ is non-zero is equivalent to the existence of a bijective map $\mathfrak{g} \in Z_{\mathfrak{f}}^{1}(G, \Gamma)$ for some $\mathfrak{f} \in \operatorname{Hom}(G, \operatorname{Aut}(\Gamma))$. Let us give two approaches to study these crossed homomorphisms. The first is to define another $\mathfrak{h} \in \operatorname{Hom}(G, \operatorname{Aut}(\Gamma))$. The idea originates from [6] and was 
formalized by the author in [19, Proposition 3.4] or [21, Proposition 2.3]. The second is to use characteristic subgroups of $\Gamma$, that is, subgroups $\Lambda$ such that $\varphi(\Lambda)=\Lambda$ for all $\varphi \in \operatorname{Aut}(\Gamma)$. The idea comes from [5] and was restated in terms of crossed homomorphisms by the author in [16, Lemma 4.1].

Proposition 2.2. Let $\mathfrak{f} \in \operatorname{Hom}(G, \operatorname{Aut}(\Gamma))$ and $\mathfrak{g} \in Z_{\mathfrak{f}}^{1}(G, \Gamma)$. Define

$$
\mathfrak{h}: G \longrightarrow \operatorname{Aut}(\Gamma) ; \quad \mathfrak{h}(\sigma)=\operatorname{conj}(\mathfrak{g}(\sigma)) \cdot \mathfrak{f}(\sigma),
$$

where $\operatorname{conj}(\cdot)=\lambda(\cdot) \rho(\cdot)$. Then:

(a) We have $\mathfrak{h} \in \operatorname{Hom}(G, \operatorname{Aut}(\Gamma))$.

(b) For any $\sigma \in G$, we have $\mathfrak{f}(\sigma)=\mathfrak{h}(\sigma)$ if and only if $\sigma \in \mathfrak{g}^{-1}(Z(\Gamma))$.

(c) The map $\sigma \mapsto \mathfrak{g}(\sigma)$ defines a homomorphism $\operatorname{ker}(\mathfrak{f}) \longrightarrow \Gamma$.

(d) The map $\sigma \mapsto \mathfrak{g}(\sigma)^{-1}$ defines a homomorphism $\operatorname{ker}(\mathfrak{h}) \longrightarrow \Gamma$.

Proof. Part (a) appeared in [19, Proposition 3.4], while parts (b) - (d) were stated in [21, Proposition 2.3]. The proofs are straightforward.

Proposition 2.3. Let $\mathfrak{f} \in \operatorname{Hom}(G, \operatorname{Aut}(\Gamma))$ and $\mathfrak{g} \in Z_{\mathfrak{f}}^{1}(G, \Gamma)$. Let $\Lambda$ be any characteristic subgroup of $\Gamma$. Consider the homomorphism

$$
\overline{\mathfrak{f}}: G \longrightarrow \operatorname{f} \operatorname{Aut}(\Gamma) \stackrel{\varphi \mapsto(\gamma \Lambda \mapsto \varphi(\gamma) \Lambda)}{\longrightarrow} \operatorname{Aut}(\Gamma / \Lambda)
$$

induced by $\mathfrak{f}$ and the map

$$
\overline{\mathfrak{g}}: G \stackrel{\mathfrak{g}}{\longrightarrow} \Gamma \stackrel{\text { quotient map }}{\longrightarrow} \Gamma / \Lambda
$$

induced by $\mathfrak{g}$. Then:

(a) We have $\overline{\mathfrak{g}} \in Z_{\overline{\mathfrak{f}}}^{1}(G, \Gamma / \Lambda)$.

(b) The preimage $\mathfrak{g}^{-1}(\Lambda)$ is a subgroup of $G$.

(c) In the case that $\mathfrak{g}$ is bijective, there is a regular subgroup of $\operatorname{Hol}(\Lambda)$ which is isomorphic to $\mathfrak{g}^{-1}(\Lambda)$.

Proof. Parts (a) and (b) are clear; see [16, Proposition 4.1] for a proof of the latter. For part (c), see [20, Proposition 3.3].

Following [5], we shall take $\Lambda$ to be a maximal characteristically subgroup of $\Gamma$. Then, the quotient $\Gamma / \Lambda$ is a non-trivial characteristically simple group, 
HOPF-GALOIS STRUCTURES ON FINITE EXTENSIONS WITH QUASISIMPLE GALOIS GROUP 5 and since $\Gamma$ is finite, we know that

$$
\Gamma / \Lambda \simeq T^{m} \text {, where } T \text { is a simple group and } m \in \mathbb{N} \text {, }
$$

in which case the structure of $\operatorname{Aut}(\Gamma / \Lambda)$ is well-known. This approach turns out to be very useful and was crucial in all of $[16,18,20,21]$.

\section{Consequences of CFSG}

In this section, let $A$ be a finite non-abelian simple group. We shall require some consequences of the classification of finite simple groups (CFSG).

One difficulty in dealing with finite quasisimple groups, as opposed to nonabelian simple groups, is that they need not be centerless. But their center is a quotient of the Schur multiplier of the associated finite non-abelian simple group; see [1, Section 33] for more on Schur multipliers.

Let $\mathfrak{m}(A)$ denote the order of the Schur multiplier of $A$. We shall say that $\mathrm{PSL}_{n}(q)$ and $\mathrm{PSU}_{n}(q)$, respectively, are non-exceptional if

$$
\mathfrak{m}\left(\mathrm{PSL}_{n}(q)\right)=\operatorname{gcd}(n, q-1) \text { and } \mathfrak{m}\left(\operatorname{PSU}_{n}(q)\right)=\operatorname{gcd}(n, q+1)
$$

As a consequence of CFSG, we know that $\mathfrak{m}(A)$ is small, in fact at most 12 , except when $A \simeq \operatorname{PSL}_{n}(q), \operatorname{PSU}_{n}(q)$. More specifically:

Lemma 3.1. Let $\mathfrak{M}=\{1,2,3,4,6,12\}$.

(a) If $A \not \operatorname{PSL}_{n}(q), \mathrm{PSU}_{n}(q)$, then $\mathfrak{m}(A) \in \mathfrak{M}$.

(b) If $A=\operatorname{PSL}_{n}(q), \mathrm{PSU}_{n}(q)$, then $\mathfrak{m}(A) \in \mathfrak{M}$ or $A$ is non-exceptional, except that $\mathfrak{m}\left(\mathrm{PSL}_{3}(4)\right)=48$ and $\mathfrak{m}\left(\mathrm{PSU}_{4}(3)\right)=36$.

Proof. See [15, Theorem 5.1.4].

Lemma 3.2. The outer automorphism group Out $(A)$ of $A$ is solvable.

Proof. This is known as Schreier conjecture; see [9, Theorem 1.46].

Lemma 3.3. Every $\varphi \in \operatorname{Aut}(A)$ has a fixed point other than $1_{A}$.

Proof. See [9, Theorem 1.48].

Lemma 3.4. There do not exist subgroups $B_{1}$ and $B_{2}$ of $A$ such that both of them have non-trivial center and $A=B_{1} B_{2}$. 
Proof. This was a conjecture of Szep and was proven in [8].

Lemma 3.5. Suppose that $A$ has a subgroup of index $p^{a}$, where $p$ is a prime and $a \in \mathbb{N}$. Then, one of the following holds:

(a) $A \simeq A_{p^{a}}$ with $p^{a} \geq 5$;

(b) $A \simeq \operatorname{PSL}_{n}(q)$ with $p^{a}=\left(q^{n}-1\right) /(q-1)$;

(c) $A \simeq \mathrm{PSL}_{2}$ (11) with $p^{a}=11$;

(d) $A \simeq M_{11}$ with $p^{a}=11$, or $A \simeq M_{23}$ with $p^{a}=23$;

(e) $A \simeq \mathrm{PSU}_{4}(2)$ with $p^{a}=27$.

Proof. See [12, Theorem 1].

\section{Proof of Theorem 1.4}

In this section, let $G$ be a finite quasisimple group, and let $N$ be any group of order $|G|$. Suppose that $e(G, N) \neq 0$, namely there is a regular subgroup $\mathcal{G}$ of $\operatorname{Hol}(N)$ isomorphic to $G$. In the next two subsections, we shall prove:

Proposition 4.1. The group $N$ must be perfect, namely $N=[N, N]$.

Proposition 4.2. The regular subgroup $\mathcal{G}$ is either $\rho(N)$ or $\lambda(N)$.

Theorem 1.4 would follow, because then $N \simeq \mathcal{G} \simeq G$.

Let us first set up the notation. By Proposition 2.1, we know that

$$
\mathcal{G}=\{\rho(\mathfrak{g}(\sigma)) \cdot \mathfrak{f}(\sigma): \sigma \in G\}, \text { where }\left\{\begin{array}{l}
\mathfrak{f} \in \operatorname{Hom}(G, \operatorname{Aut}(N)), \\
\mathfrak{g} \in Z_{\mathfrak{f}}^{1}(G, N) \text { is bijective. }
\end{array}\right.
$$

Alternatively, we may rewrite it as

$$
\mathcal{G}=\left\{\lambda(\mathfrak{g}(\sigma))^{-1} \cdot \mathfrak{h}(\sigma): \sigma \in G\right\}, \text { where } \mathfrak{h} \in \operatorname{Hom}(G, \operatorname{Aut}(N))
$$

is defined as in Proposition 2.2. Let $M$ be any maximal characteristic subgroup of $N$. Then, as in Proposition 2.3, we have homomorphisms

$$
\overline{\mathfrak{f}}, \overline{\mathfrak{h}}: G \longrightarrow \operatorname{f,h} \longrightarrow \operatorname{Aut}(N) \stackrel{\varphi \mapsto(\eta M \mapsto \varphi(\eta) M)}{\longrightarrow} \operatorname{Aut}(N / M)
$$

induced by $\mathfrak{f}$ and $\mathfrak{h}$, respectively, and a surjective crossed homomorphism

$$
\overline{\mathfrak{g}}: G \stackrel{\mathfrak{g}}{\longrightarrow} \mathrm{\text {quotientmap }} \longrightarrow N / M
$$


HOPF-GALOIS STRUCTURES ON FINITE EXTENSIONS WITH QUASISIMPLE GALOIS GROUP 7 with respect to $\overline{\mathfrak{f}}$ induced by $\mathfrak{g}$. We shall also need the facts that $Z(G)$ is a quotient of the Schur multiplier of $G / Z(G)$, all proper normal subgroups of $G$ are contained in $Z(G)$.

See $[1,(33.8)]$ for the former, and the latter is an easy exercise.

4.1. Non-perfect groups. Suppose for contradiction that $N$ is not perfect, in which case we may take $M$ to contain $[N, N]$. By $(2.1)$, we then have

$$
N / M \simeq(\mathbb{Z} / p \mathbb{Z})^{m}, \text { where } p \text { is a prime and } m \in \mathbb{N} \text {. }
$$

Let us first make a simple observation.

Lemma 4.3. The homomorphism $\overline{\mathfrak{f}}$ is non-trivial and $m \geq 2$.

Proof. Suppose for contradiction that $\overline{\mathfrak{f}}$ is trivial. Then, the map

$$
G \stackrel{\overline{\mathfrak{g}}}{\longrightarrow} N / M \stackrel{\simeq}{\longrightarrow}(\mathbb{Z} / p \mathbb{Z})^{m}
$$

is a homomorphism by Proposition $2.2(\mathrm{c})$, and so must be trivial because $G$ is perfect. This contradicts that $\overline{\mathfrak{g}}$ is surjective, so indeed $\overline{\mathfrak{f}}$ is non-trivial. It follows that $m \geq 2$, for otherwise

$$
G \stackrel{\overline{\mathfrak{f}}}{\longrightarrow} \operatorname{Aut}(N / M) \stackrel{\simeq}{\longrightarrow}(\mathbb{Z} / p \mathbb{Z})^{\times}
$$

would be trivial again because $G$ is perfect, which we know is impossible.

Define $H=\mathfrak{g}^{-1}(M)$, which is a subgroup of $G$ by Proposition 2.3(b), and whose order is $|M|$ because $\mathfrak{g}$ is bijective. Thus, we have $[G: H]=p^{m}$. Put

$$
p^{z}=[H Z(G): H]=[Z(G): H \cap Z(G)] \text {, where } 0 \leq z \leq m .
$$

Note that $p$ divides $|Z(G)|$ unless $z=0$. Also $m-z \geq 1$, for otherwise

$$
G=H Z(G) \text { and in particular } G=[G, G]=[H, H],
$$

which is impossible because $H$ is a proper subgroup. Since

$$
\left[\frac{G}{Z(G)}: \frac{H Z(G)}{Z(G)}\right]=\frac{[G: H]}{[H Z(G): H]}=p^{m-z},
$$

by Lemma 3.5 one of the following holds: 
(a) $G / Z(G) \simeq A_{p^{m-z}}$ with $p^{m-z} \geq 5$;

(b) $G / Z(G) \simeq \operatorname{PSL}_{n}(q)$ with $p^{m-z}=\left(q^{n}-1\right) /(q-1)$;

(c) $G / Z(G) \simeq \mathrm{PSL}_{2}(11)$ with $p^{m-z}=11$;

(d) $G / Z(G) \simeq M_{11}$ with $p^{m-z}=11$, or $G / Z(G) \simeq M_{23}$ with $p^{m-z}=23$;

(e) $G / Z(G) \simeq \operatorname{PSU}_{4}(2)$ with $p^{m-z}=27$.

Since Theorem 1.4 holds when $G$ is a finite non-abelian simple group by [5], and when $G$ is the double cover of $A_{n}$ with $n \geq 5$ by [16, Theorem 1.6], we may henceforth assume that:

Assumption. The center $Z(G)$ of $G$ is non-trivial.

Recall from (4.1) that $|Z(G)|$ divides $\mathfrak{m}(G / Z(G))$. Hence, this assumption in particular restricts that $\mathfrak{m}(G / Z(G)) \neq 1$.

Assumption. The group $G$ is not the double cover of an alternating group.

Lemma 4.4. We must be in case (b).

Proof. Case (d) does not occur by our first assumption because

$$
\mathfrak{m}\left(M_{11}\right)=1=\mathfrak{m}\left(M_{23}\right) .
$$

To deal with cases (a), (c), and (e), note that

$$
\mathfrak{m}\left(A_{n}\right)=\left\{\begin{array}{ll}
2 & \text { if } n=5 \text { or } n \geq 8 \\
6 & \text { if } n=6,7
\end{array} \text { and } \mathfrak{m}\left(\mathrm{PSL}_{2}(11)\right)=2=\mathfrak{m}\left(\mathrm{PSU}_{4}(2)\right)\right.
$$

For case (a), we must have $p^{m-z}=7$ by our second assumption. For case (c), we have $p^{m-z}=11$. In both cases, note that $p$ does not divide $\mathfrak{m}(G / Z(G))$, so $z=0$ and $m=1$. But this contradicts Lemma 4.3. For case (e), we have $p^{m-z}=27$. Since $p$ does not divide $\mathfrak{m}(G / Z(G))$, we have $z=0$ and $m=3$. Also, by our first assumption, necessarily

$$
|Z(G)|=2 \text {, and so }|G|=2\left|\operatorname{PSU}_{4}(2)\right|=51840 .
$$

But $\left|\mathrm{GL}_{m}(p)\right|=\left|\mathrm{GL}_{3}(3)\right|=11232$, so the homomorphism

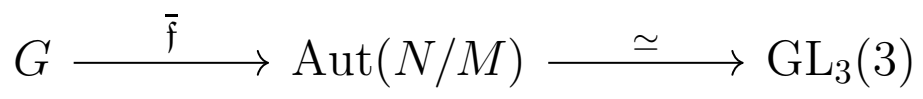


HOPF-GALOIS STRUCTURES ON FINITE EXTENSIONS WITH QUASISIMPLE GALOIS GROUP 9 is trivial by (4.2) and by comparing orders. But this contradicts Lemma 4.3. Thus, indeed we must be in case (b).

In view of Lemma 4.4, we now know that

$$
G / Z(G) \simeq \operatorname{PSL}_{n}(q) \text { with } p^{m-z}=\left(q^{n}-1\right) /(q-1)
$$

By [15, Theorem 5.1.4], we also know that $\mathfrak{m}\left(\operatorname{PSL}_{n}(q)\right)=\operatorname{gcd}(n, q-1)$, unless $(n, q)$ equals one of the five pairs stated in the next lemma. Let us first rule out these cases.

Lemma 4.5. We have $(n, q) \neq(2,4),(2,9),(3,2),(3,4),(4,2)$.

Proof. Suppose for contradiction that $(n, q)$ is one of the stated pairs. Then this pair must be $(2,4)$ or $(3,2)$ because $\left(q^{n}-1\right) /(q-1)$ is a prime power. Note that

$$
p^{m-z}=\frac{q^{n}-1}{q-1}= \begin{cases}5 & \text { if }(n, q)=(2,4) \\ 7 & \text { if }(n, q)=(3,2) .\end{cases}
$$

But $\mathfrak{m}\left(\mathrm{PSL}_{2}(4)\right)=2=\mathfrak{m}\left(\mathrm{PSL}_{3}(2)\right)$. In either case, since $p$ does not divide 2 , we see that $z=0$, and so $m=1$. But this contradicts Lemma 4.3.

Lemma 4.6. We have $G \simeq \mathrm{SL}_{n}(q)$ and $|Z(G)|=n=p$.

Proof. By Lemma 4.5 and our first assumption, respectively, we have

$$
\mathfrak{m}(G / Z(G))=\operatorname{gcd}(n, q-1) \text { and } \mathfrak{m}(G / Z(G)) \neq 1
$$

As noted in $[12,(3.3)]$, that $p^{m-z}=\left(q^{n}-1\right) /(q-1)$ implies that $n$ is a prime. It then follows that

$$
\operatorname{gcd}(n, q-1)=n, \text { and so } q \equiv 1(\bmod n)
$$

Moreover, we must have $|Z(G)|=n$ and also $G \simeq \operatorname{SL}_{n}(q)$, the universal cover of $\mathrm{PSL}_{n}(q)$. Note that

$$
p^{m-z} \equiv q^{n-1}+\cdots+q+1 \equiv 1+\cdots+1+1 \equiv n \equiv 0(\bmod n)
$$

so in fact $n=p$. This completes the proof.

We shall now use the next proposition to get a contradiction and thus prove Proposition 4.1; cf. [5, Theorem 4.3] and the argument in [5, Section 4]. 
Proposition 4.7. Suppose that $\mathrm{SL}_{n}(q)$ has a non-trivial irreducible representation of degree $d$ over a field of characteristic coprime to $q$, where

$$
(n, q) \neq(3,2),(3,4),(4,2),(4,3) \text {. }
$$

Then, we have

$$
d \geq \begin{cases}(q-1) / \operatorname{gcd}(2, q-1) & \text { if } n=2, \\ \left(q^{n}-1\right) /(q-1)-2 & \text { if } n \geq 3 .\end{cases}
$$

Proof. See [13, Lemma 9.1.1 and Theorem 9.1.5].

Proof of Proposition 4.1. By Lemmas 4.3 and 4.6, we have $m \geq 2$ and there is a non-trivial homomorphism

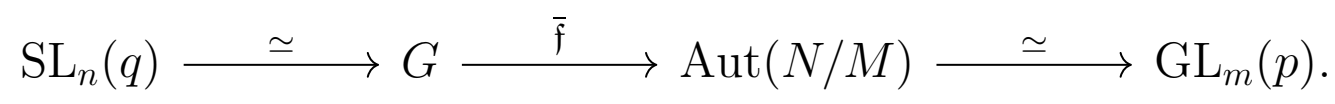

Since $p^{m-z}=\left(q^{n}-1\right) /(q-1)$, we have $\operatorname{gcd}(p, q)=1$ and also $(n, q) \neq(4,3)$. By Lemma 4.5, we may then apply Proposition 4.7 as follows. Recall that $|Z(G)|=n=p$ by Lemma 4.6, which in turn implies $z=0,1$.

For $n=2$, note that $p^{m-z}=q+1$, and we obtain

$$
m \geq \frac{q-1}{\operatorname{gcd}(2, q-1)}=\frac{p^{m-z}-2}{\operatorname{gcd}\left(2, p^{m-z}-2\right)} \geq \frac{2^{m-1}-2}{2} .
$$

For $n \geq 3$, similarly we have

$$
m \geq \frac{q^{n}-1}{q-1}-2=p^{m-z}-2 \geq 3^{m-1}-2 .
$$

From the above inequalities, we deduce that

$$
(m, p)= \begin{cases}(2,2),(3,2),(4,2) & \text { if } n=2 \\ (2,3) & \text { if } n \geq 3\end{cases}
$$

Since $n^{m-z}=p^{m-z}=\left(q^{n}-1\right) /(q-1)$ and $z=0,1$, it follows that

$$
(n, q)=(2,3),(2,7), \text { and in fact necessarily }(n, q)=(2,7)
$$

because $\mathrm{PSL}_{2}(3)$ is not simple. We are left with the possibility $G \simeq \mathrm{SL}_{2}(7)$.

Using the Holomorph and RegularSubgroups commands in Magma [2], we checked that $\operatorname{Hol}(N)$ has no regular subgroup isomorphic to $G \simeq \mathrm{SL}_{2}(7)$ 
HOPF-GALOIS STRUCTURES ON FINITE EXTENSIONS WITH QUASISIMPLE GALOIS GROUP for all non-perfect groups $N$ of order 336. We remark that in fact it suffices to check the insolvable groups $N$ of order 336 by [20, Theorem 1.10]. Thus, we obtain a contradiction, so indeed $N$ must be perfect.

4.2. Perfect groups. By Proposition 4.1, we know that $N$ must be perfect, in which case all quotients of $N$ are also perfect. By (2.1), we then have

$$
N / M \simeq T^{m}, \text { where } T \text { is non-abelian simple and } m \in \mathbb{N},
$$

and by [5, Lemma 3.2] for example, we know that

$$
\operatorname{Aut}\left(T^{m}\right)=\operatorname{Aut}(T)^{m} \rtimes S_{m}
$$

We shall also use Burnside's theorem, which states that the order of a finite insolvable group is divisible by at least three distinct primes.

The cases $G / Z(G) \simeq \operatorname{PSL}_{n}(q), \operatorname{PSU}_{n}(q)$ require special arguments because then $\mathfrak{m}(G / Z(G))$ and so $|Z(G)|$ could be arbitrarily large. Let us recall that

$$
\begin{aligned}
& \left|\mathrm{PSL}_{n}(q)\right|=\frac{1}{\operatorname{gcd}(n, q-1)}\left(q^{\left(\begin{array}{c}
n \\
2
\end{array}\right)} \prod_{i=2}^{n}\left(q^{i}-1\right)\right), \\
& \left|\mathrm{PSU}_{n}(q)\right|=\frac{1}{\operatorname{gcd}(n, q+1)}\left(q^{\left(\begin{array}{c}
n \\
2
\end{array}\right)} \prod_{i=2}^{n}\left(q^{i}-(-1)^{i}\right)\right) .
\end{aligned}
$$

We shall prove that either $\mathfrak{f}$ or $\mathfrak{h}$ is trivial in a sequence of steps.

Lemma 4.8. The image $\overline{\mathfrak{f}}(G)$ lies in $\operatorname{Inn}(N / M)$.

Proof. Below, we shall show that the homomorphism

$$
\overline{\mathfrak{f}}_{S_{m}}: G \stackrel{\overline{\mathfrak{f}}}{\longrightarrow} \operatorname{Aut}(N / M) \stackrel{\text { identification }}{=} \operatorname{Aut}(T)^{m} \rtimes S_{m} \stackrel{\text { projection }}{\longrightarrow} S_{m}
$$

is trivial. Then, the image $\overline{\mathfrak{f}}(G)$ lies in $\operatorname{Aut}(T)^{m}$, and the homomorphism

$$
G \stackrel{\bar{f}}{\longrightarrow} \operatorname{Aut}(T)^{m} \stackrel{\text { projection }}{\longrightarrow} \operatorname{Out}(T)^{m}
$$

is also trivial, because $G$ is perfect while $\operatorname{Out}(T)$ is solvable by Lemma 3.2. It follows that $\overline{\mathfrak{f}}(G)$ lies in $\operatorname{Inn}(T)^{m}$, which is identified with $\operatorname{Inn}(N / M)$.

To prove that $\overline{\mathfrak{f}}_{S_{m}}$ is trivial, let $\ell$ be any prime factor of $|T|$. For any finite group $\Gamma$, let $v_{\ell}(\Gamma)$ be the non-negative integer such that $\ell^{v_{\ell}(\Gamma)}$ exactly divides 
$|\Gamma|$. We have $v_{\ell}(G) \geq m$ because $|G|=|N|=|T|^{m}|M|$. It is well-known that

$$
v_{\ell}\left(S_{m}\right)=\left\lfloor\frac{m}{\ell}\right\rfloor+\left\lfloor\frac{m}{\ell^{2}}\right\rfloor+\left\lfloor\frac{m}{\ell^{3}}\right\rfloor+\cdots \text { and so } v_{\ell}\left(S_{m}\right)<\frac{m}{\ell-1} .
$$

Since $G / \operatorname{ker}\left(\overline{\mathfrak{f}}_{S_{m}}\right)$ embeds into $S_{m}$, we then deduce that

$$
v_{\ell}(G)-v_{\ell}\left(\operatorname{ker}\left(\overline{\mathfrak{f}}_{S_{m}}\right)\right) \leq v_{\ell}\left(S_{m}\right)<\frac{m}{\ell-1} .
$$

Suppose now for contradiction that $\overline{\mathfrak{f}}_{S_{m}}$ is non-trivial, in which case $\operatorname{ker}\left(\overline{\mathfrak{f}}_{S_{m}}\right)$ lies in $Z(G)$ by (4.2). From $m \leq v_{\ell}(G)$ and (4.4), we see that

$$
m-v_{\ell}(Z(G)) \leq v_{\ell}(G)-v_{\ell}(Z(G)) \leq v_{\ell}(G)-v_{\ell}\left(\operatorname{ker}\left(\overline{\mathfrak{f}}_{S_{m}}\right)\right)<\frac{m}{\ell-1},
$$

and so $v_{\ell}(Z(G)) \geq 1$. This implies that every prime factor of $|T|$ also divides $|Z(G)|$. From Burnside's theorem and (4.1), it then follows that $\mathfrak{m}(G / Z(G))$ has at least three distinct prime divisors. From Lemma 3.1, we deduce that

$G / Z(G) \simeq \operatorname{PSL}_{n}(q), \operatorname{PSU}_{n}(q)$ with $\operatorname{PSL}_{n}(q), \operatorname{PSU}_{n}(q)$ non-exceptional.

Put $v_{\ell}(G)=x$ and $v_{\ell}(Z(G))=y$, where $x, y \geq 1$. Then, we have

$$
x-y<\frac{m}{\ell-1} \leq \frac{x}{\ell-1} \text { and in particular } y>\frac{\ell-2}{\ell-1} \cdot x .
$$

Also, from (3.1) and (4.1), we see that

$$
|Z(G)| \text { divides } \begin{cases}\operatorname{gcd}(n, q-1) & \text { if } G / Z(G) \simeq \operatorname{PSL}_{n}(q), \\ \operatorname{gcd}(n, q+1) & \text { if } G / Z(G) \simeq \operatorname{PSU}_{n}(q) .\end{cases}
$$

Since $\ell^{y}$ divides $|Z(G)|$, from (4.5) we have $\ell^{y} \leq n$, that is $y \leq \log (n) / \log (\ell)$. Observe that the order formulae in (4.3) imply that

$$
\begin{gathered}
(q-1)^{n-2} \text { divides }\left|\mathrm{PSL}_{n}(q)\right|, \\
(q+1)^{\lfloor n / 2\rfloor-1} \text { divides }\left|\mathrm{PSU}_{n}(q)\right| .
\end{gathered}
$$

Since $\ell^{y}$ divides $|Z(G)|$, again from (4.5) we see that $\ell$ divides $q-1$ and $q+1$, respectively, and in particular

$$
v_{\ell}(G)-v_{\ell}(Z(G)) \geq \begin{cases}n-2 & \text { if } G / Z(G) \simeq \operatorname{PSL}_{n}(q) \\ \left\lfloor\frac{n}{2}\right\rfloor-1 & \text { if } G / Z(G) \simeq \operatorname{PSU}_{n}(q)\end{cases}
$$


HOPF-GALOIS STRUCTURES ON FINITE EXTENSIONS WITH QUASISIMPLE GALOIS GROUP

In either case, this in turns yields

$$
x-1 \geq x-y \geq \frac{n}{2}-\frac{1}{2}-1 \text { and so } x \geq \frac{n-1}{2} .
$$

Again, by Burnside's theorem, we may take $\ell \geq 5$. We obtain

$$
\frac{4}{3} \cdot \frac{\log (n)}{\log (5)} \geq \frac{\ell-1}{\ell-2} \cdot \frac{\log (n)}{\log (\ell)} \geq \frac{\ell-1}{\ell-2} \cdot y>x \geq \frac{n-1}{2} .
$$

But then $n=2$, which contradicts that $5 \leq \ell^{y} \leq n$. Hence, indeed $\overline{\mathfrak{f}}_{S_{m}}$ must be trivial, and this completes the proof.

Lemma 4.9. We have $N / M \simeq T$.

Proof. We have $\operatorname{Inn}(N / M) \simeq N / M \simeq T^{m}$. Depending on whether $\overline{\mathfrak{f}}$ is trivial or not, by Proposition 2.2(c) and Lemma 4.8, respectively, we see that there is a non-trivial homomorphism $\varphi: G \longrightarrow T^{m}$. Let $1 \leq i \leq m$ be such that

$$
\left.\varphi^{(i)}: G \stackrel{\varphi}{\longrightarrow} T^{m} \stackrel{\text { projection }}{\longrightarrow} T^{(i)} \text { (the } i \text { th copy of } T\right)
$$

is non-trivial. Since $\operatorname{ker}\left(\varphi^{(i)}\right)$ lies in $Z(G)$ by $(4.2)$, we have

$$
\frac{|T|^{m}|M|}{|Z(G)|}\left[Z(G): \operatorname{ker}\left(\varphi^{(i)}\right)\right]=\frac{|G|}{\left|\operatorname{ker}\left(\varphi^{(i)}\right)\right|}=\left|\varphi^{(i)}(G)\right|=\frac{|T|}{\left[T^{(i)}: \varphi^{(i)}(G)\right]},
$$

and in particular

$$
|Z(G)|=|T|^{m-1}|M|\left[Z(G): \operatorname{ker}\left(\varphi^{(i)}\right)\right]\left[T^{(i)}: \varphi^{(i)}(G)\right] .
$$

Suppose for contradiction that $m \geq 2$, in which case $|T|$ divides $|Z(G)|$ and hence $\mathfrak{m}(G / Z(G))$ by (4.1). Since all groups of order at most 48 are solvable, from Lemma 3.1 and (3.1), we see that

$$
G / Z(G) \simeq \operatorname{PSL}_{n}\left(\ell^{a}\right), \operatorname{PSU}_{n}\left(\ell^{a}\right) \text {, where } \ell \text { is a prime, and } \ell \nmid|Z(G)| \text {. }
$$

But $\ell$ divides $|G|=|T|^{m}|M|$ by (4.3) and thus $|T||M|$. This shows that (4.6) cannot hold, so indeed $m=1$, and we have $N / M \simeq T$.

For any $\sigma \in G$, recall that $\overline{\mathfrak{h}}(\sigma)=\operatorname{conj}(\overline{\mathfrak{g}}(\sigma)) \cdot \overline{\mathfrak{f}}(\sigma)$ by definition, and so

$$
\overline{\mathfrak{f}}(\sigma)=\overline{\mathfrak{h}}(\sigma) \Longleftrightarrow \overline{\mathfrak{g}}(\sigma)=1_{N / M} \Longleftrightarrow \sigma \in \mathfrak{g}^{-1}(M)
$$

because $N / M$ has trivial center. 
Lemma 4.10. We have $G / Z(G) \simeq T$ and $|M|=|Z(G)|$.

Proof. By Lemma 4.8, the image $\overline{\mathfrak{f}}(G)$ lies in $\operatorname{Inn}(N / M)$, and so plainly $\overline{\mathfrak{h}}(G)$ lies in $\operatorname{Inn}(N / M)$ as well. Since $\operatorname{Inn}(N / M) \simeq N / M$, we then have

$$
|G / \operatorname{ker}(\overline{\mathfrak{f}})|,|G / \operatorname{ker}(\overline{\mathfrak{h}})| \leq|N / M|, \text { and so }|M| \leq|\operatorname{ker}(\overline{\mathfrak{f}})|,|\operatorname{ker}(\overline{\mathfrak{h}})|
$$

Trivially $\overline{\mathfrak{f}}(\sigma)=\overline{\mathfrak{h}}(\sigma)$ for all $\sigma \in \operatorname{ker}(\overline{\mathfrak{f}}) \cap \operatorname{ker}(\overline{\mathfrak{h}})$, so by (4.7) we have

$$
|\operatorname{ker}(\overline{\mathfrak{f}}) \cap \operatorname{ker}(\overline{\mathfrak{h}})| \leq\left|\mathfrak{g}^{-1}(M)\right|, \text { and }\left|\mathfrak{g}^{-1}(M)\right|=|M|
$$

because $\mathfrak{g}$ is bijective. Since $\overline{\mathfrak{g}}$ is surjective, we also have the factorization

$$
\operatorname{Inn}(N / M)=\overline{\mathfrak{f}}(G) \overline{\mathfrak{h}}(G) \text {, whence } \overline{\mathfrak{f}}(G) \text { or } \overline{\mathfrak{h}}(G) \text { has trivial center }
$$

by Lemmas 3.4 and 4.9. This implies that $\operatorname{ker}(\overline{\mathfrak{f}}) \subset \operatorname{ker}(\overline{\mathfrak{h}})$ or $\operatorname{ker}(\overline{\mathfrak{h}}) \subset \operatorname{ker}(\overline{\mathfrak{f}})$ has to hold, for otherwise $\operatorname{ker}(\overline{\mathfrak{f}}), \operatorname{ker}(\overline{\mathfrak{h}}) \lesseqgtr Z(G)$ by $(4.2)$, and both $\overline{\mathfrak{f}}(G)$ and $\overline{\mathfrak{h}}(G)$ would have non-trivial center. By symmetry, we may assume that the former inclusion holds. Then, from the above inequalities, we deduce that

$$
|M|=|\operatorname{ker}(\overline{\mathfrak{f}})|, \text { and so } G / \operatorname{ker}(\overline{\mathfrak{f}}) \simeq \operatorname{Inn}(N / M) \simeq T
$$

by comparing orders. But $\operatorname{ker}(\overline{\mathfrak{f}})$ lies in $Z(G)$ again by $(4.2)$, and $T$ has trivial center, so in fact $\operatorname{ker}(\overline{\mathfrak{f}})=Z(G)$. Both claims now follow.

Lemma 4.11. Either $\overline{\mathfrak{f}}$ or $\overline{\mathfrak{h}}$ is trivial, and $Z(G)=\mathfrak{g}^{-1}(M)$.

Proof. Suppose for contradiction that both $\overline{\mathfrak{f}}$ and $\overline{\mathfrak{h}}$ are non-trivial. By (4.2), this means that both $\operatorname{ker}(\overline{\mathfrak{f}})$ and $\operatorname{ker}(\overline{\mathfrak{h}})$ lie in $Z(G)$. Since $G / \operatorname{ker}(\overline{\mathfrak{f}}), G / \operatorname{ker}(\overline{\mathfrak{h}})$ embed into $\operatorname{Inn}(N / M)$, by comparing orders and by Lemma 4.10 , we have

$$
\operatorname{ker}(\overline{\mathfrak{f}})=Z(G)=\operatorname{ker}(\overline{\mathfrak{h}}) \text { and } \overline{\mathfrak{f}}(G)=\operatorname{Inn}(N / M)=\overline{\mathfrak{h}}(G) .
$$

From (4.7), we then deduce that $Z(G) \subset \mathfrak{g}^{-1}(M)$, which must be an equality by Lemma 4.10 and the bijectivity of $\mathfrak{g}$. The above also implies that $\overline{\mathfrak{f}}$ and $\overline{\mathfrak{h}}$, respectively, induce isomorphisms

$$
\varphi_{f}, \varphi_{h}: G / Z(G) \longrightarrow \operatorname{Inn}(N / M) \text {, and } \varphi_{h}^{-1} \circ \varphi_{f} \in \operatorname{Aut}(G / Z(G)) .
$$

But for any $\sigma \in G$, again by (4.7), we have

$$
\left(\varphi_{h}^{-1} \circ \varphi_{f}\right)(\sigma Z(G))=\sigma Z(G) \Longleftrightarrow \sigma \in \mathfrak{g}^{-1}(M) \Longleftrightarrow \sigma Z(G)=1_{G / Z(G)} .
$$


This contradicts Lemma 3.3. Thus, at least one of $\overline{\mathfrak{f}}$ or $\overline{\mathfrak{h}}$ is trivial.

Now, by Proposition 2.2(c),(d), the surjective map

$$
\varphi: G \longrightarrow N / M ; \quad \varphi(\sigma)= \begin{cases}\overline{\mathfrak{g}}(\sigma) & \text { if } \overline{\mathfrak{f}} \text { is trivial } \\ \overline{\mathfrak{g}}(\sigma)^{-1} & \text { if } \overline{\mathfrak{h}} \text { is trivial }\end{cases}
$$

is a homomorphism, and $\operatorname{ker}(\varphi)$ lies in $Z(G)$ by (4.2). By comparing orders, we see from Lemma 4.10 that in fact $\operatorname{ker}(\varphi)=Z(G)$. But in both cases, we have $\operatorname{ker}(\varphi)=\mathfrak{g}^{-1}(M)$ by definition, so the claim follows.

Lemma 4.12. We have $M \subset Z(N)$.

Proof. Since $M$ is normal in $N$, we have a homomorphism

$$
\Phi: N \stackrel{\eta \mapsto\left(x \mapsto \eta x \eta^{-1}\right)}{\longrightarrow} \operatorname{Aut}(M) \stackrel{\text { projection }}{\longrightarrow} \operatorname{Out}(M)
$$

whose kernel clearly contains $M$. Either $\Phi$ is trivial or $\operatorname{ker}(\Phi)=M$ because $N / M$ is simple by Lemma 4.9 .

Suppose first that $\Phi$ is trivial. This implies that

$$
N=M C, \text { where } C \text { is the centralizer of } M \text { in } N .
$$

Given $i \in \mathbb{N}_{\geq 0}$ and a group $\Gamma$, let $\Gamma^{(i)}$ denote its $i$ th derived subgroup. Since elements in $M$ and $C$ commute, we easily see that

$$
N^{(i)}=M^{(i)} C^{(i)} \text { for all } i \in \mathbb{N}_{\geq 0} .
$$

By Lemma 4.11 and Proposition 2.3(c), there is a regular subgroup of $\operatorname{Hol}(M)$ which is isomorphic to $Z(G)$. Since $Z(G)$ is abelian, it then follows from [20, Theorem 1.3(b)] that $M$ is metabelian, namely $M^{(2)}=1$. Since $N$ is perfect, we deduce that

$$
N=N^{(1)}=N^{(2)}=M^{(2)} C^{(2)}=C^{(2)} \text { and so } N=C .
$$

This means that all elements in $N$ centralize $M$, that is $M \subset Z(N)$.

Suppose now that $\operatorname{ker}(\Phi)=M$, in which case $N / M$ embeds into $\operatorname{Out}(M)$. From Lemmas 4.9 and 4.10, we then see that

$$
G / Z(G) \text { embeds into } \operatorname{Out}(M) \text {, and recall }|M|=|Z(G)| \text {. }
$$


Notice that then $\operatorname{Out}(M)$ and in particular $\operatorname{Aut}(M)$ must be insolvable. Let $\mathfrak{M}=\{1,2,3,4,6,12\}$ be as in Lemma 3.1. We consider three cases.

1. $\mathfrak{m}(G / Z(G))$ lies in $\mathfrak{M}$ : By (4.1) we know that $|M|=|Z(G)|$ divides one of the numbers in $\mathfrak{M}$. But we checked in MAGMA [2] that no such group $M$ has insolvable $\operatorname{Aut}(M)$.

2. $G / Z(G) \simeq \operatorname{PSL}_{3}(4), \mathrm{PSU}_{4}(3)$ : Recall Lemma 3.1. Again by (4.1) we know that $|M|=|Z(G)|$ divides 48 or 36 . Since $\operatorname{Aut}(M)$ must be insolvable, we checked in Magma that $M$ has SmallGroup ID equal to one of

$$
(8,5),(16,14),(24,15),(48,50),(48,51),(48,52),
$$

and in particular $\mathfrak{m}(G / Z(G))$ is divisible by 8 . Hence, we have

$$
G / Z(G) \simeq \operatorname{PSL}_{3}(4), \text { and note that }\left|\mathrm{PSL}_{3}(4)\right|=20160 \text {. }
$$

Again, using the OuterOrder command, we computed in MAGmA that

$$
|\operatorname{Out}(M)|=168,20160,336,120,1344,40320 \text {, }
$$

respectively, when $M$ has SmallGroup ID in (4.8). Moreover, the group $M$ is abelian and there is no subgroup isomorphic to $\mathrm{PSL}_{3}(4)$ in $\operatorname{Aut}(M)$, when $M$ has SmallGroup ID equal to $(16,14),(48,52)$. We then deduce that $G / Z(G)$ cannot embed into $\operatorname{Out}(M)$.

3. $G / Z(G) \simeq \operatorname{PSL}_{n}(q), \operatorname{PSU}_{n}(q)$ with $\operatorname{PSL}_{n}(q), \operatorname{PSU}_{n}(q)$ non-exceptional: We may assume that $M \neq 1$. Then, by [14, Corollary 3.3], we have

$$
|\varphi| \leq|M|-1 \text { for all } \varphi \in \operatorname{Aut}(M) .
$$

Since $|M|=|Z(G)|$, from (3.1) and (4.1), we deduce that

$$
|\varphi \operatorname{Inn}(M)| \leq|\varphi| \leq \min \{n-1, q\} \text { for all } \varphi \in \operatorname{Aut}(M) .
$$

Since $\operatorname{Aut}(M)$ is insolvable, we must have $n \geq 4$, and so $n=2+1+n_{0}$ for some integer $n_{0} \geq 1$. But then $G / Z(G)$ would contain an element of order $q^{2}-1>q$ by $[3$, Corollary $3(3)]$ and so cannot embed into $\operatorname{Out}(M)$.

In all cases, we obtained a contradiction. Hence, the case $\operatorname{ker}(\Phi)=M$ in fact does not occur, so indeed $M \subset Z(N)$. 
We are now ready to prove Proposition 4.2.

Proof of Proposition 4.2. By Lemma 4.11, either $\overline{\mathfrak{f}}$ or $\overline{\mathfrak{h}}$ is trivial. Since $N$ is perfect, and $M \subset Z(N)$ by Lemma 4.12, the homomorphism

$$
\operatorname{Aut}(N) \longrightarrow \operatorname{Aut}(N / M) ; \quad \varphi \mapsto(\eta M \mapsto \varphi(\eta) M)
$$

is injective; see the proof of [16, Proposition 3.5(c)], for example. Therefore, either $\mathfrak{f}$ or $\mathfrak{h}$ is trivial. But clearly

$$
\mathcal{G}= \begin{cases}\rho(N) & \text { if } \mathfrak{f} \text { is trivial } \\ \lambda(N) & \text { if } \mathfrak{h} \text { is trivial }\end{cases}
$$

This completes the proof.

\section{ACKNOWLEDGMENTS}

Research supported by the Young Scientists Fund of the National Natural Science Foundation of China (Award No.: 11901587).

The author thanks the referee for helpful comments.

\section{REFERENCES}

[1] M. Aschbacher, Finite group theory. Second edition. Cambridge Studies in Advanced Mathematics, 10. Cambridge University Press, Cambridge, 2000.

[2] W. Bosma, J. Cannon, and C. Playoust, The Magma algebra system. I. The user language, J. Symbolic Comput., 24 (1997), 23-265.

[3] A. A. Buturlakin, Spectra of finite linear and unitary groups (Russian), Algebra Logika 47 (2008), no. 2, 157-173, 264; translation in Algebra Logic 47 (2008), no. 2, 91-99.

[4] N. P. Byott, Uniqueness of Hopf-Galois structure of separable field extensions, Comm. Algebra 24 (1996), no. 10, 3217-3228. Corrigendum, ibid. no. 11, 3705.

[5] N. P. Byott, Hopf-Galois structures on field extensions with simple Galois groups, Bull. London Math. Soc. 36 (2004), no. 1, 23-29.

[6] S. Carnahan and L. N. Childs, Counting Hopf-Galois structures on non-abelian Galois field extensions, J. Algebra 218 (1999), no. 1, 81-92.

[7] L. N. Childs, Taming wild extensions: Hopf algebras and local Galois module theory. Mathematical Surveys and Monographs, 80. American Mathematical Society, Providence, RI, 2000.

[8] E. Fisman and Z. Arad, A proof of Szep's conjecture on nonsimplicity of certain finite groups, J. Algebra 108 (1987), no. 2, 340-354.

[9] D. Gorenstein, Finite simple groups. An introduction to their classification. University Series in Mathematics. Plenum Publishing Corp., New York, 1982.

[10] C. Greither and B. Pareigis, Hopf-Galois theory for separable field extensions, J. Algebra 106 (1987), no. $1,261-290$. 
[11] L. Guarnieri and L. Vendramin, Skew braces and the Yang-Baxter equation, Math. Comp. 86 (2017), no. 307, 2519-2534.

[12] R. M. Guralnick, Subgroups of prime power index in a simple group, J. Algebra 81 (1983), no. 2, 304-311.

[13] R. Guralnick, T. Penttila, C. E. Praeger, and J. Saxl, Linear groups with orders having certain large prime divisors, Proc. London Math. Soc. (3) 78 (1999), no. 1, 167-214.

[14] I. M. Isaacs, Finite group theory. Graduate Studies in Mathematics, 92. American Mathematical Society, Providence, RI, 2008.

[15] P. Kleidman and M. Liebeck, The subgroup structure of the finite classical groups. London Mathematical Society Lecture Note Series, 129. Cambridge University Press, Cambridge, 1990.

[16] C. Tsang, Non-existence of Hopf-Galois structures and bijective crossed homomorphisms, J. Pure Appl. Algebra 223 (2019), no. 7, 2804-2821.

[17] C. Tsang, Hopf-Galois structures of isomorphic-type on a non-abelian characteristically simple extension, Proc. Amer. Math. Soc. 147 (2019), no. 12, 5093-5103.

[18] C. Tsang, Hopf-Galois structures on a Galois $S_{n}$-extension, J. Algebra 531 (2019), 349-361.

[19] C. Tsang, On the multiple holomorph of a finite almost simple group, New York J. Math. 25 (2019), 949-963.

[20] C. Tsang and C. Qin, On the solvability of regular subgroups in the holomorph of a finite solvable group, Internat. J. Algebra Comput. 30 (2020), no. 2, 253-265.

[21] C. Tsang, Hopf-Galois structures on finite extensions with almost simple Galois group, J. Number Theory 214 (2020), 286-311.

School of Mathematics (Zhuhai), Sun Yat-Sen University, Zhuhai, Guangdong, China

E-mail address: zengshy26@mail.sysu.edu.cn

$U R L:$ http://sites.google.com/site/cindysinyitsang/ 